\title{
A quantitative analysis of the diurnal evolution of Ionospheric Alfvén resonator magnetic resonance features and calculation of changing IAR parameters
}

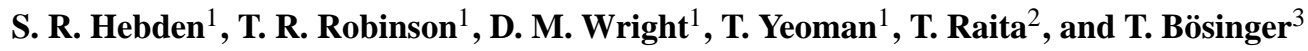 \\ ${ }^{1}$ Dept. of Phys. and Astronomy, Univ. of Leicester, Univ. Road, Leicester, LE1 7RH, UK \\ ${ }^{2}$ Sodankylä Geophysical Observatory, Tähteläntie 62, 99600 Sodankylä, Finland \\ ${ }^{3}$ Dept. of Phys. Sciences, Div. of Physics, Sect. of Space Phys. Res., P. O. Box 3000, 90014 University of Oulu, Finland
}

Received: 29 September 2004 - Revised: 6 April 2005 - Accepted: 8 April 2005 - Published: 28 July 2005

\begin{abstract}
Resonance features of the Ionospheric Alfvén Resonator (IAR) can be observed in pulsation magnetometer data from Sodankylä, Finland using dynamic spectra visualizations. IAR resonance features were identified on 13 of 30 days in October 1998, with resonance structures lasting for 3 or more hours over 10 intervals. The diurnal evolution of the harmonic features was quantified for these 10 intervals using a manual cursor-clicking technique. The resonance features displayed strong linear relationships between harmonic frequency and harmonic number for all of the time intervals studied, enabling a homogeneous cavity model for the IAR to be adopted to interpret the data. This enabled the diurnal variation of the effective size of the IAR to be obtained for each of the 10 time intervals. The average effective size was found to be $530 \mathrm{~km}$, and to have an average variation of $32 \%$ over each time interval: small compared to the average variation in Alfvén velocity of $61 \%$. Thus the diurnal variation of the harmonics is chiefly caused by the changing plasma density within the IAR due to changing insolation. This study confirms Odzimek (2004) that the dominating factor affecting the IAR eigenfrequencies is the variation in the Alfvén velocity at the F-layer ion-density peak, with the changing IAR size affecting the IAR eigenfrequencies to a smaller extent. Another IAR parameter was derived from the analysis of the IAR resonance features associated with the phase matching structure of the standing waves in the IAR. This parameter varied over the time intervals studied by $20 \%$ on average, possibly due to changing ionospheric conductivity.
\end{abstract}

Keywords. Ionosphere (Auroral ionosphere; Wave propagation) - Radio science (Electromagnetic noise and interference)

Correspondence to: S. R. Hebden

(sophiehebden@yahoo.com)

\section{Introduction}

The Ionospheric Alfvén Resonator (IAR) is the term given to the vertical structure associated with the decay in plasma density going from the ionosphere to the magnetosphere, whose existence was first predicted by Polyakov, (1976). Alfvén waves propagating along the geomagnetic field lines are partially reflected from regions of large Alfvén velocity gradients. The lower boundary region occurs in the ionospheric F-layer. The upper boundary of the IAR corresponds to the region where the Alfvén waves of interest undergo partial reflection at the large Alfvén velocity gradient caused by the rapid decrease in plasma density above the F-layer density peak, whilst the geomagnetic field strength decreases with altitude more slowly.

IAR resonance features at high latitudes are observable in the electromagnetic spectra between 0.2 and $5 \mathrm{~Hz}$ (The frequency range of Pc1 waves) (Belyaev et al., 1999). These eigenfrequencies of the IAR were first observed in 1985 by Belyaev et al. (1987) in the form of multiple horizontally banded spectral resonance structures (SRS) in frequencytime plots of mid-latitude magnetic background noise between $0.1 \mathrm{~Hz}$ and $10 \mathrm{~Hz}$ derived from pulsation magnetometer data. They were observed at high latitudes $(L \sim 6$, where $\mathrm{L}$-shell value relates to latitude as $\mathrm{L}=\cos ^{-2} \lambda_{E}$ ) by Belyaev et al. (1999).

IAR resonance features are manifested in the form of multiple maxima and minima in the magnetic field power spectrum which are quite stable, often lasting for several hours until fading out or being masked by more intense wave activity. The modulation depth of the power spectrum can be up to $50 \%$, and the frequency interval $\Delta f$ between the peaks is generally in the range $0.5-3 \mathrm{~Hz}$ at mid-latitudes (Belyaev et al., 2000). They are regularly observed at low and mid latitudes in high time resolution magnetometer data, especially at night-time. Belyaev et al. (1990) observed diurnal variations of resonance peak characteristics in one of the 
two orthogonal components of the tangential magnetic field at the Earth's surface at the mid latitude station of Nizhny Novgorod $(\mathrm{L} \sim 2.6)$. The diurnal variation in frequency and spacing was proportional to $1 / f_{0} F 2$, where $f_{0} F 2$ is the critical frequency of the F2-layer in the ionosphere. The resonance peaks increased in modulation depth, frequency and spacing towards the maximum at midnight and decreased towards dawn, with resonance features rarely observed during the day. These authors attributed the characteristics of the spectral resonance features to the changing parameters of the IAR.

Hickey et al. (1996) extended the observation at Nizhny Novgorod by reporting detection of banded magnetic spectra from an $\mathrm{L} \sim 1.5$ station in California. The first evidence for the IAR at high latitudes (Belyaev et al., 1999) was in the form of resonance features observed on 4 out of 12 nights of data collection using the sensitive magnetometer experiment at Kilpisjärvi, Finland in November 1993. These authors qualitatively compared the frequency evolution of the resonance features with the change in ionospheric electron density from EISCAT (Rishbeth and Williams, 1985) incoherent scatter radar data. Resonance features were reported by Demekhov et al. (2000) to be "a regular feature" at the high latitude magnetometer station at Sodankylä, Finland $(L \sim 5.1)$. These authors presented 4 days of observations from the stations at Nizhny Novgorod and Sodankylä, Finland, and used a model of the IAR (published in Izvestiya vuzov-Radiofizika by Belyaev et al. in 1989) to make quantitative comparisons of the predicted spectra with the magnetic features. They found that the diurnal variation of the spectral resonance features could be well explained by the variation in the local ionospheric parameters.

More recently Yahnin et al. (2003) used continuous observations from 1995-1999 at the Sodankylä magnetometer station to define the morphology of the IAR resonance features at high latitudes. These authors found the occurrence rate $(P)$ of resonance features to be higher in the nighttime than the daytime, and higher in winter than in summer. They made a statistical study on the frequency spacing of the IAR eigenfrequencies $(\Delta f)$ and of $P$, and showed that their diurnal, seasonal and long-term behaviour agreed well with IAR theory predictions based on numerical calculations of the reflection coefficient at the top-side ionosphere. These authors concluded that observations of SRS could be used for estimation of upper ionosphere parameters and for improving the existing ionosphere models.

In this paper, the evolution of the resonant frequencies with local time is quantified for days when they were clearly visible. Spectra from a whole month of pulsation magnetometer data from Sodankylä, Finland are considered. To interpret the data, a simple 1-D vertical model was adopted (Trakhtengerts et al., 2000) in which the IAR consists of 3 layers of constant density. The middle layer represents the standing wave cavity which is bounded below by a conduction layer and above by a low density layer leading to the wave trapping and resonance features of the IAR. Data from a co-located ionosonde were combined with the IAR reso- nance feature analysis results to calculate IAR model parameters which correspond to the effective IAR cavity size and the phase matching structure of the standing wave in the IAR cavity.

This study confirms the earlier results that the diurnal evolution of the IAR resonance features is caused by the evolution of the local IAR parameters via detailed day-by-day spectral analysis. It also illustrates the need for experimental investigation of the reflection of Alfvén waves at the lower boundary region of the ionosphere and how this relates to the ionospheric conductivity. Although this study is inconclusive on this point due to a lack of incoherent scatter radar data when IAR resonance features were observed in October 1998, it shows that linear relationships between the frequency $f$ and harmonic number $N$ of resonance features enable the reflection characteristics of Alfvén waves at the lower boundary to be investigated using the simple IAR model.

\section{Experimental data and processing method}

October 1998 was chosen for this study of the structure of the IAR because during this month the University of Leicester ran some modulated ionospheric heating experiments at the Troms $\varnothing$ HF heating facility (Stubbe et al., 1982) to coincide with overhead passes of the FAST satellite (Carlson et al., 1998). In one of the heating experiments, narrow-band electric and particle flux signatures were observed by the satellite for about $4 \mathrm{~s}$ (Robinson et al., 2000, Kolesnikova et al., 2002, Cash et al., 2002, Wright et al., 2003). This occurred when the satellite traversed magnetic flux tubes that map down to the heated ionospheric patch. The data were best explained by the presence of the IAR with an upper boundary $\sim 850 \mathrm{~km}$ above the satellite altitude (Cash et al., 2002). Thus any additional evidence for the existence of the IAR at similar latitudes in October 1998 makes the IAR interpretation of the FAST satellite data more convincing. By studying a month of data it is possible to ascertain how frequently IAR resonance features can be observed, and the common ionospheric conditions occurring during the observations. There are currently several pulsation magnetometers located in northern Scandinavia (see Fig. 1), but only data from the Sodankylä (SOD) pulsation magnetometer station $\left(67.40^{\circ} \mathrm{N}, 26.60^{\circ} \mathrm{E}\right.$ geographic coordinates) were available with high accuracy timing for October 1998.

Figure 2 provides an example of typical magnetic IAR resonance features seen during the month of October 1998. It shows a 12-h colour dynamic power spectrum on 4th October 1998 for the linear east-west (or D component) polarization of differential magnetic field. Power spectral density is shown in colour scale normalized to a reference frequency of $3 \mathrm{~Hz}$. This means that the maximum power occurring in the $3 \mathrm{~Hz}$ bin sets the maximum for the colour scale (maximum=red). This is plotted as a function of hour of the day ( $x$ axis) and frequency ( $y$ axis). A lower threshold of $40 \%$ of the peak power was chosen because this produced the clearest 
Finnish pulsation magnetometer chain

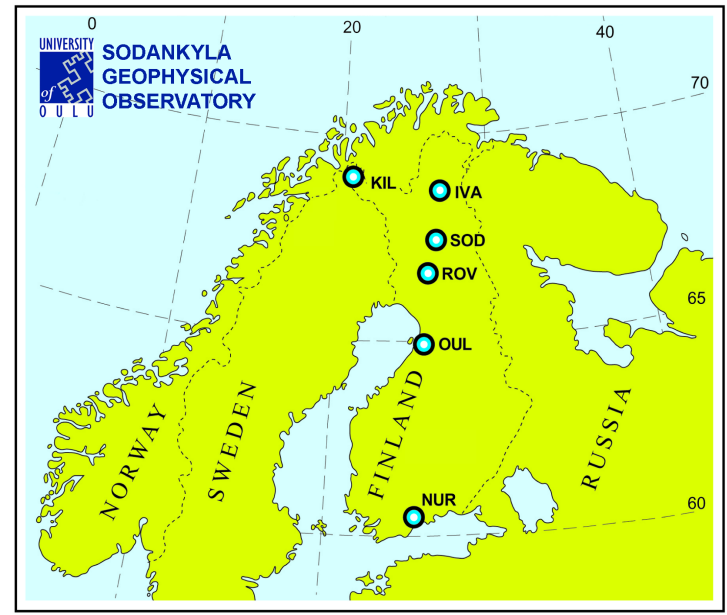

Fig. 1. The Finnish pulsation magnetometer chain (courtesy of SGO).

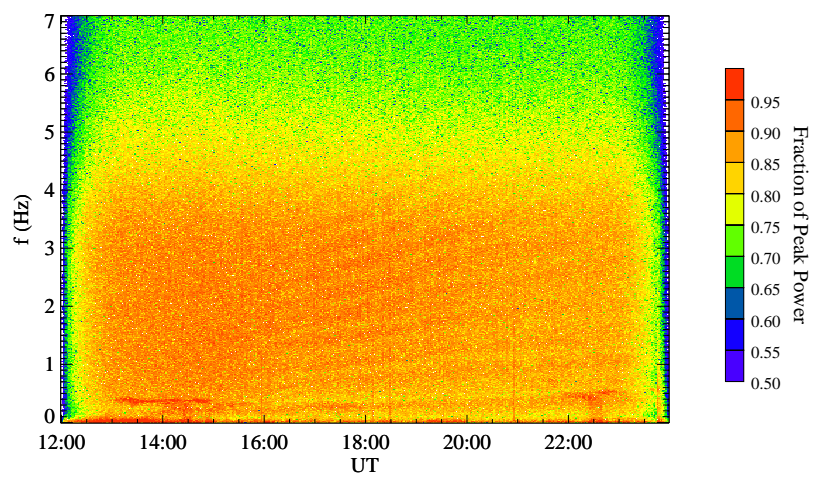

Fig. 2. Pulsation magnetometer data for 04/10/98 from Sodankylä, Finland displayed as colour dynamic power spectral plot for the east west (D component) polarisation. The window and slip size are $100 \mathrm{~s}$; power spectral density is normalised to peak power at $3 \mathrm{~Hz}$ and not calibrated.

resonance features, below the threshold a spectral component is omitted from the plot. The dynamic spectrum was produced by applying a Fast Fourier Transform (FFT) to a sliding window of $100 \mathrm{~s}$, and moving it along by increments of $100 \mathrm{~s}$ along the differential magnetic field time series. Thus the frequency resolution is $0.01 \mathrm{~Hz}$ and the time resolution is $100 \mathrm{~s}$. At each time-slip along the data series, the DC component in the data within the FFT window is removed, then the window is convolved with a standard Hanning window (Lynn, 1984) to minimise the number of terms in the frequency response of the window. This is effectively a "raised cosine bell" function applied to $10 \%$ of the data points at each edge of the window.

The magnetic data used throughout this paper were not calibrated for the frequency response of the magnetometer because the sensitivity of the search coil sensors increases with frequency up to $3 \mathrm{~Hz}$. This is an advantage because the weak IAR resonance peaks around $3 \mathrm{~Hz}$ are easier to observe,

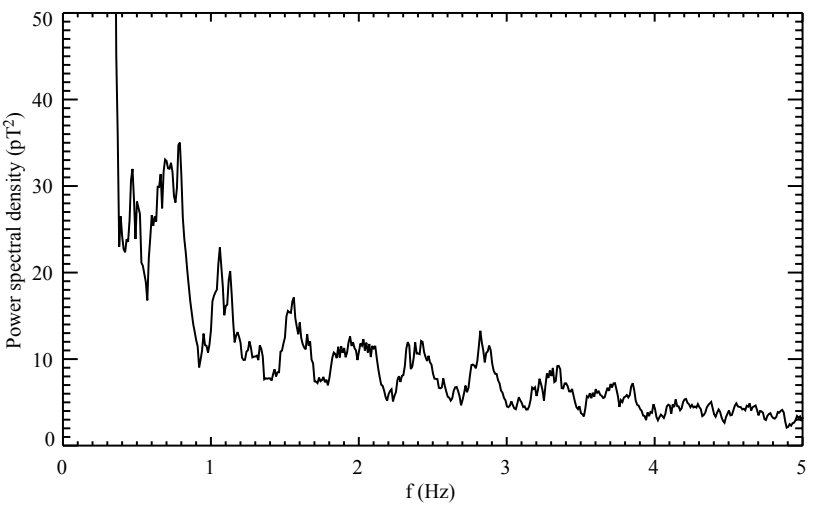

Fig. 3. Power spectral density for 18:00-18:40 UT from pulsation magnetometer at Sodankylä, Finland on 4 October 1998 for window and slip size of $100 \mathrm{~s}$. This is the average of 24 power spectra, smoothed with a boxcar of width 3 .

whilst the magnetometer is less sensitive to the naturally high amplitude Pc1 waves at about $0.3 \mathrm{~Hz}$.

With reference to Fig. 2, the resonance features appear at about 16:00 UT as 9-10 coloured horizontal bands roughly equally spaced in frequency, and slowly increasing in frequency and frequency spacing $(\Delta f)$ with time towards midnight. The remaining linear north-south (H component) and circular polarization components (RH and LH components which are not shown) occur at the same frequencies as the east-west component displayed in Fig. 2, but are of slightly lower amplitude, not distinguishable as resonance features until 18:00 UT. The power spectral density in the D component is $\sim 10 \mathrm{pT}^{2} / \mathrm{Hz}$ from $0-3 \mathrm{~Hz}$ at 18:00 UT (see Fig. 3), but it varies in time differently for each polarization. However, it is possible to use the persistence in time of the resonance features to detect their presence in the data. Pc1 waves can be seen at almost all times in Fig. 2 at frequencies below $0.5 \mathrm{~Hz}$. However, true IAR resonance features (with more than one harmonic) are observable in the $\mathrm{D}$ component from about 16:00 UT until midnight. The modulation depth between peaks and troughs is best determined from a single calibrated differential power spectrum, shown for the D component in Fig. 3 for 18:00-18:40 UT on 4th October 1998. This plot is the average of 24 individual spectra of $100 \mathrm{~s}$ of data, thus it has a time resolution of $40 \mathrm{~min}$ and frequency resolution of $0.01 \mathrm{~Hz}$. Averaging over 24 spectra removes the ephemeral features in the data leaving the coherent resonance features. It can be seen that the modulation depth between peaks and troughs is $\sim 5-10 \mathrm{pT}^{2} / \mathrm{Hz}$ in the range 0 $3 \mathrm{~Hz}$, or $\sim 50 \%$ of the peak power at this time.

Dynamic spectra were plotted for every day in October except 16th October 1998 when there were data gaps. Resonance features of the IAR in the form of multiple harmonic bands that evolve in frequency with time were observed on 13 days. Considering the geomagnetic activity during October, resonance features were observed on 9 out of 10 days when the average daily $\mathrm{A}_{p}<5$, and only 4 out of 20 days when $A_{p} \geq 5$. The 3rd October 1998 had the max- 


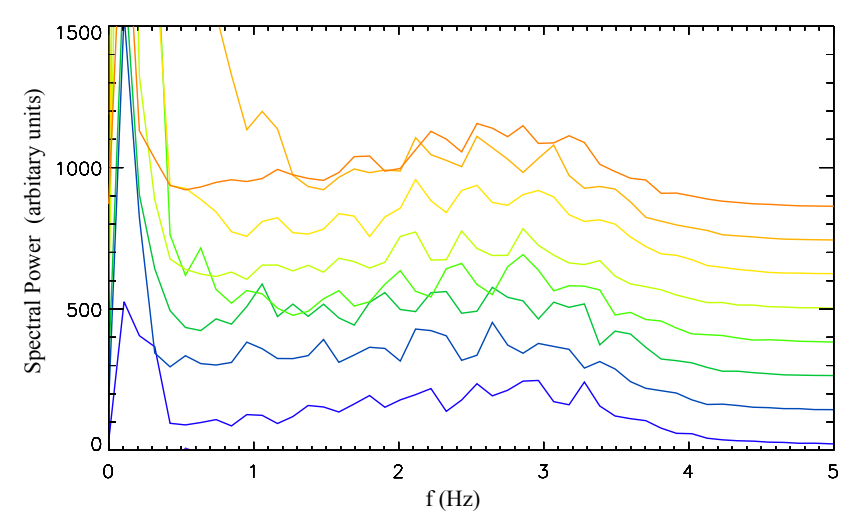

Fig. 4. Stack plot of 20 min-averaged power spectra for the east west (D component) polarisation magnetic field on 4 October 1998 from pulsation magnetometer at Sodankylä, Finland. The spectra are from 16:40-19:00 UT going from purple to orange with increasing time.

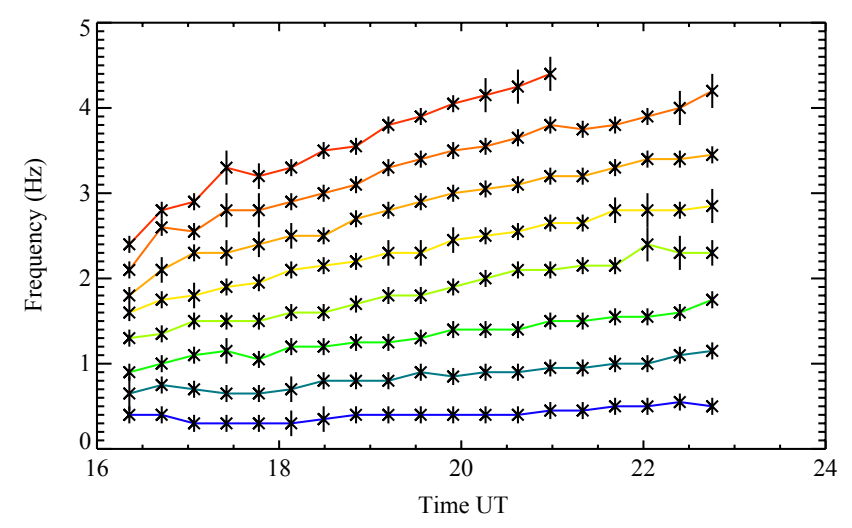

Fig. 5. Spectral resonance features on 4 October 1998 for the north south (H component) polarisation magnetic field from Sodankylä, Finland. The frequency variation over time for the first 8 harmonics was derived using averaged spectra, which represent $20 \mathrm{~min}$ of data. The error bars give the uncertainty in frequency of the resonance peaks.

imum average daily $\mathrm{A}_{p}$ on which resonance features were observed. For this day the average $\mathrm{A}_{p}$ was 13.6, although the resonance features occurred during the afternoon when the $A_{p}$ index dropped to 3 or 4 . This corresponded with the end of ULF wave activity, and a flattening out of the spectra after midday. For the 7 days in October 1998 with disturbed magnetospheric conditions $\left(\mathrm{A}_{p}>20\right)$, IAR resonance features were never observed since there was intense, highly variable ULF wave activity flooding the spectra and masking the background noise in which the resonance features appear.

Quantifying the diurnal frequency variation of the IAR resonance features is difficult because of the low modulation depth in the harmonic peaks at any instance in time. In attempts to find a quick and accurate technique to quantify the SRS features, two different manual methods were developed that exploit the coherence between consecutive spectra. An automated method was used to compare the change in $\Delta f$ with time and thus to validate the manual method used for the data analysis.

\subsection{Method 1}

The first method was based on the spectral resonance feature visualisation used by Belyaev et al. (1999). Each polarization was processed by taking an FFT of a 10-s window of data and moving the window along by $10 \mathrm{~s}$, and then averaging over 120 spectra. This produced spectra like Fig. 3 with a time resolution of $20 \mathrm{~min}$ and a frequency resolution of $0.1 \mathrm{~Hz}$, which were displayed as stack plots to show the diurnal evolution of the resonance features. Figure 4 is an example of a series of uncalibrated spectra for $\mathrm{D}$ component polarization on 4th October 1998 from 16:40-19:00 UT. The IAR resonance peaks are more easily identifiable in the context of a few hours of stack plots because they are extremely coherent in time. The peak frequencies, their amplitudes and uncertainties were determined for each 20 min spectrum, at each polarization using the on-screen interactive cursor clicking technique. An average of the four polarizations was taken to give the most accurate diurnal evolution of the spectral resonance features. An example of the variation in frequency with time for 8 harmonics in the afternoon of 4th October 1998 for the $\mathrm{H}$ component polarization is shown in Fig. 5, with the error bars given by the uncertainty in judging the peak frequencies. The frequency spacing between peaks increased by $0.21 \mathrm{~Hz}$ from 17:00-22:00 UT.

\subsection{Method 2}

To determine the variation in the frequency spacing between harmonics $(\Delta f)$ with time, an automated method was used that relies on the regular spacing of IAR harmonics in the magnetic power spectra. This method treats the stack plots of spectra used in method 1 as mini time series of data containing a fixed period wave (since harmonics of the IAR are equally spaced in frequency). FFTs of these spectra were performed to obtain "pseudo spectra". A peak in a pseudo spectrum indicates that there are multiple harmonics present in the real spectrum, and the inverse of the "pseudo frequency" of the peak gives $\Delta f$. Figure 6 columns 2 and 4 illustrate the change of the pseudo frequency peak with time. Time increases by 20 min per panel going from top to bottom. The main peak decreases in pseudo frequency with time, thus the pseudo wavelength increases. Therefore the spacing of the peaks $(\Delta f)$ in the real spectra increases by $0.20 \mathrm{~Hz}$; this matches the value obtained using method 1 to within $0.01 \mathrm{~Hz}$. Thus this method confirms the result from method 1 for 17:00-22:00 UT on 4th October 1998.

\subsection{Method 3}

The third technique allows the diurnal evolution of the harmonic frequencies to be quantified (only frequency spacing is quantified using the automated method) more quickly than method 1 and with greater accuracy as a consequence of the 20 min time resolution of each spectral plot for method 1 . 
Method 3 used dynamic spectra for 12-h sections of data; visualisations like that displayed in Fig. 2. These were plotted on the computer screen for each of the four polarization components at maximum pixel resolution so that the resonance features could be identified interactively with the cursor. Where a harmonic peak was visible above the background level, the variation in frequency with time was defined by about 10 interactive cursor clicks. The long extent in time of the dynamic spectra aided identification of the horizontally banded pattern within the context of the 12-h period. Least squares regression lines were found from all of the cursor clicks for each harmonic, and the average found for all four polarizations. The standard deviations of the harmonic cursor points about the lines of best fit provide information about the uncertainty involved in the method, and hence the uncertainty in the frequency of each harmonic with time. This process is illustrated in Fig. 7 for the resonance features on 4th October 1998. Panel a) shows where interactive cursor clicks (represented by black diamonds) are applied along the first 8 harmonics in the dynamic spectrum for the $\mathrm{H}$ component polarization. Panel b) is a frequency versus time plot of the cursor points for 8 harmonics at all four polarizations, with the $\mathrm{H}$ component in red, D in yellow, $\mathrm{RH}$ in green and LH in blue. Panel c) shows the least squares regression lines for each harmonic through the combined polarization cursor points. Their uncertainty is indicated by the dotted lines either side of the solid lines, which are one standard deviation from the least squares regression lines. Method 3 yielded an increase in $\Delta f$ of $0.20 \mathrm{~Hz}$ from 17:00-22:00 UT. The agreement of this with methods 1 and 2 validates it as an appropriate method for the task of quantifying the diurnal variation of the spectral resonance features.

Resonance features observed over 10 time intervals in October 1998 were analyzed in detail using method 3. These 10 were chosen because at least 3 harmonics were visible in the dynamic colour spectra for at least $3 \mathrm{~h}$. Spectral features that do not exhibit at least 3 regularly spaced harmonics may not be caused by wave resonance in the IAR, and time intervals of less than $3 \mathrm{~h}$ are unsuitable for detailed analysis of the development of the IAR over time.

\subsection{Ionosonde measurements}

Hourly $f_{0} F 2$ data from the ionosonde at Sodankylä were obtained for days on which magnetic spectral resonance features occurred. These data sets, co-located with the magnetometer, were used to calculate the diurnal variation of the peak electron densities in the $\mathrm{F} 2$ region of the ionosphere assuming that solar illumination is the main driver of ionospheric plasma density variations. It is reasonable to assume that the density within the IAR varies in the same way as the density at the bottom of the IAR, given by the F2 peak, and that this can be used to calculate the diurnal evolution of the Alfvén velocity in the IAR which dominates the evolution of the IAR eigenfrequencies (adopted by Demekhov et al., 2000, Yahnin et al., 2003) (this is shown in the next section). Thus the analysis of the IAR resonance features in the mag-
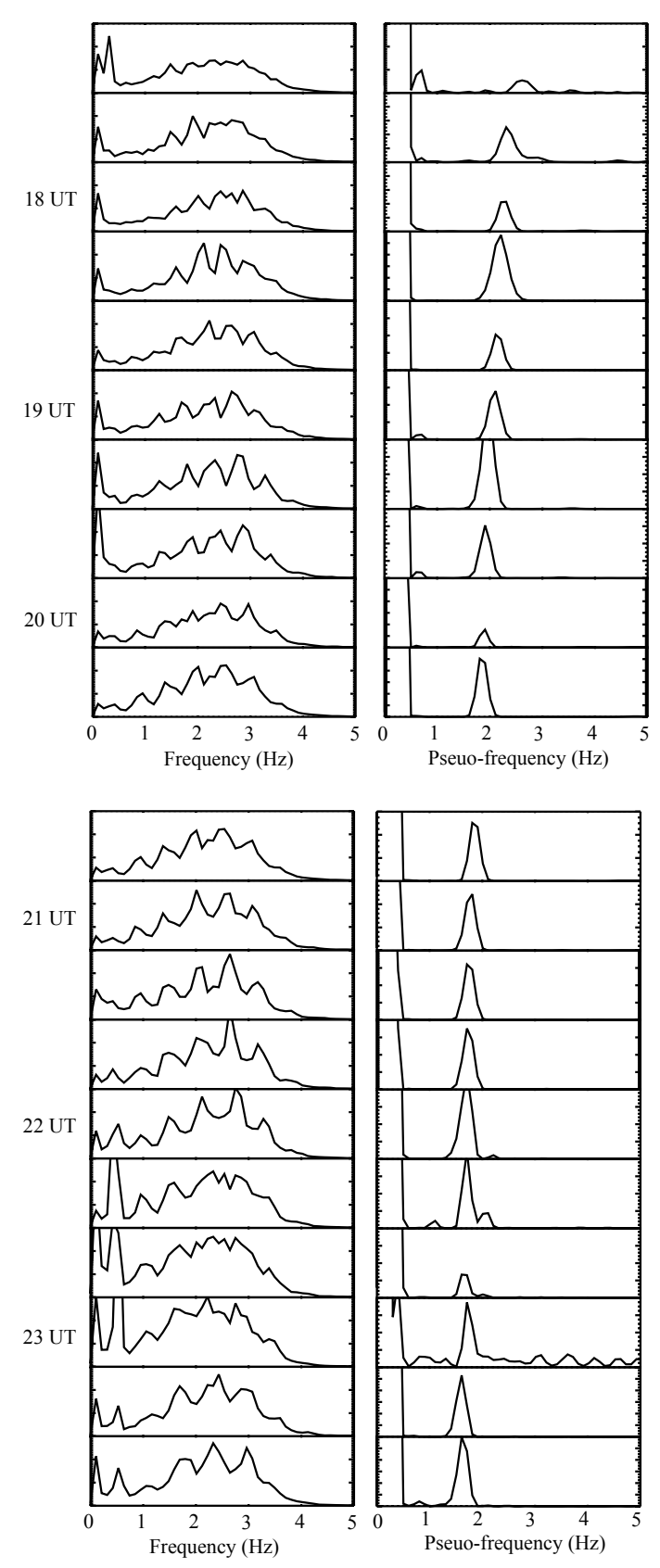

Fig. 6. Columns on the left side of the diagram show the $20 \mathrm{~min}$ averaged power spectral density for the east west ( $\mathrm{H}$ component) polarisation magnetic field variation at Sodankylä on 4 October 1998. The plots show magnetic field power spectral density (arbitrary amplitude) as a function of frequency. Columns on the right show the Fast Fourier Transforms of these power spectra, such that the peak in pseudo-frequency indicates the periodicity in frequency in columns 1 and 3 due to the Alfvén wave harmonics resonating within the IAR. The peak in pseudo-frequency decreases from $\sim 2.4-1.7 \mathrm{~Hz}$ from 17:00-22:00 UT. This indicates that the frequency spacing of the IAR harmonics increases over the time interval from $0.4-0.6 \mathrm{~Hz}$.

netic spectra, combined with the ionosonde data, allowed the changing IAR parameters to be determined (see Sect. 3). 
a)

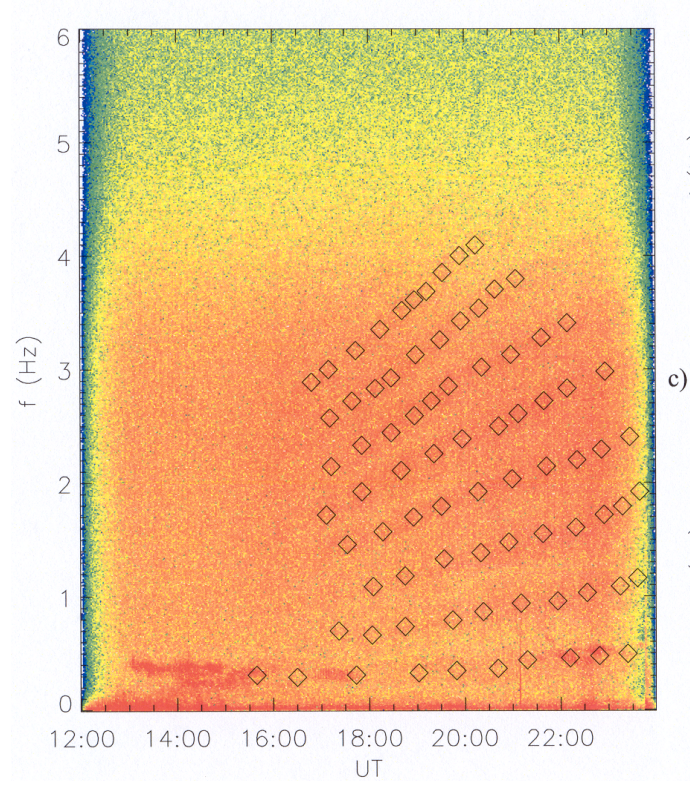

b)
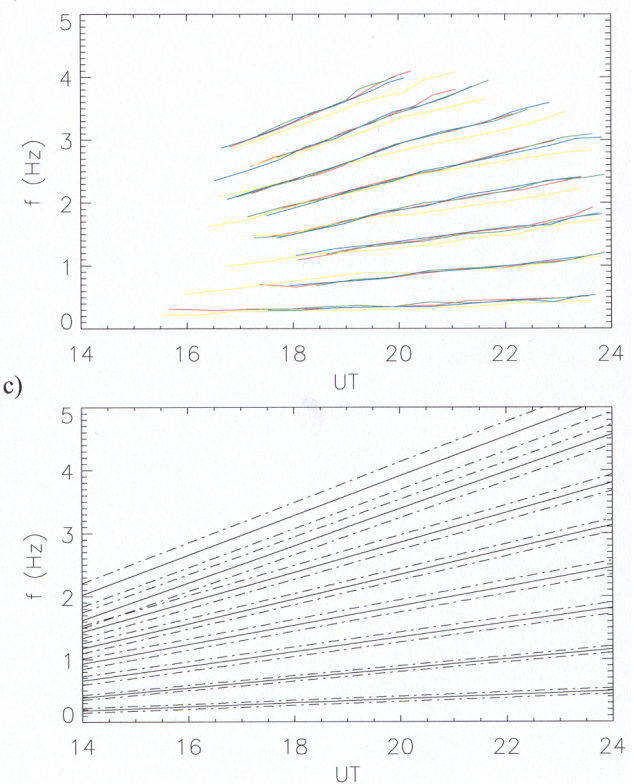

Fig. 7. Panel (a) shows pulsation magnetometer data from Sodankylä, Finland for 4 October 1998 in the north south (H component) polarisation displayed as a colour dynamic power spectral plot. The black diamonds represent cursor clicks used for quantifying the diurnal evolution of the resonance features. Panel(b) shows the cursor clicked resonance features for all the polarisations on the same date with north south component (red), east west (yellow), right-handed circular (green) and left-handed circular polarisation (blue). Panel (c) shows the lines of best fit through the cursor points for each polarisation. The dot-dashed error margins are given by the standard deviation of the points about each line.

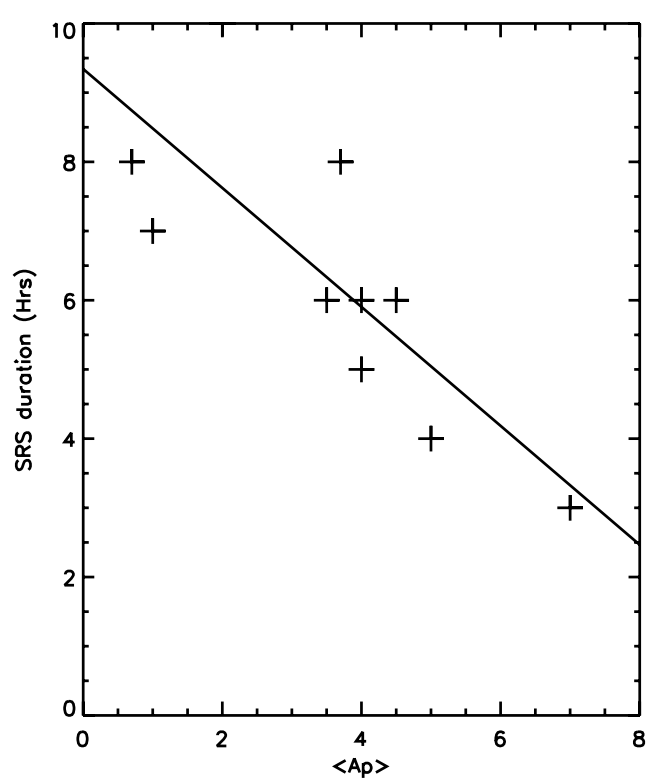

Fig. 8. Scatter plot and least squares regression line for data collected at Sodankylä, Finland during October 1998. Duration of the IAR resonance features observed in the pulsation magnetometer data is plotted against the mean $\mathrm{A}_{p}$ during the observation.

\section{Results and discussions}

Table 1 is a summary of the resonance feature analysis for the 10 time intervals studied from October 1998. Columns 1-6 show dates of observation and the approximate observation times (in UT) of the resonance features in the magnetic dynamic spectra, the number of harmonics seen, the mean $\mathrm{A}_{p}$, the range of $f_{0} F 2$ critical frequency values during the interval, and the harmonic spacing $(\Delta f)$ during these times respectively. Columns 7-9 give model IAR parameters derived from data analysis which will be explained later. All except one morning interval from 02:00-06:00 LT on 4 October 1998 are night-time observations of increasing harmonic frequencies and spacing, typically starting from 18:00 LT and fading out after 02:00 LT. Generally more harmonics were visible on days when the average $\mathrm{A}_{p}$ during the observation period was low, since the total power in the spectra was lower, making small amplitude signals detectable at the higher frequency end of the spectra despite the drop-off in sensitivity of the magnetometer. The resonance features were observed for longer when the $\mathrm{A}_{p}$ was small; a quiet magnetosphere is best for observing relatively weak IAR resonance features. This is illustrated in Fig. 8, which is a scatter plot of the duration of the resonance feature observations against the mean $\mathrm{A}_{p}$ during this time. A least squares regression line is plotted, whose product moment correlation coefficient (Pearson's r) was good ( -0.79$)$, indicating a negative relationship between resonance feature duration and mean $\mathrm{A}_{p}$. 
Table 1. Summary of the IAR resonance feature analysis for October 1998. The 2nd column shows the approximate times between which IAR spectral resonance features were observed in the pulsation magnetometer data from Sodankylä, Finland. The 3rd column gives the number of harmonics that were clearly visible in the dynamic colour spectra. Column 4 shows the average $\mathrm{A}_{p}$ index during the resonance feature observation. The 5 th and 6 th columns indicate how the $f_{0} F 2$ critical frequency measured by the ionosonde at Sodankylä and the resonance feature spacing varied from the start to finish of the resonance features. Column 7 gives the average product moment correlation coefficient over the resonance feature observations. This is the mean of the hourly correlations between resonance peak frequency and harmonic number. The 8th and 9th columns indicate the range of values of $\Phi$ and $L$ calculated for each time interval, and their associated errors.

\begin{tabular}{lccccccll}
\hline Date & Time (UT) & $N$ & Mean A $_{p}$ & $f o F 2(\mathrm{MHz})$ & $\Delta f(\mathrm{~Hz})(2$. d.p. $)$ & Mean $r$ & $\Phi(2 . \mathrm{d} . \mathrm{p})$. & $L(\mathrm{~km})$ \\
\hline 3 October 1998 & $16: 00-24: 00$ & 3 & 3.7 & $2.4-5.8$ & $0.47-0.84$ & 0.9916 & $0.50-0.55 \pm 0.15$ & $430-630 \pm 80$ \\
4 October 1998 & $00: 00-05: 00$ & 4 & 4 & $1.8-2.1$ & $0.84-0.72$ & 0.9993 & $0.78-0.80 \pm 0.07$ & $700-1140 \pm 100$ \\
4 October 1998 & $16: 00-24: 00$ & 8 & 2 & $3.8-7.7$ & $0.26-0.65$ & 0.9995 & $0.65-0.73 \pm 0.15$ & $420-680 \pm 50$ \\
5 October 1998 & $18: 00-24: 00$ & 7 & 4.5 & $3.0-6.5$ & $0.41-0.69$ & 0.9994 & $0.52-0.55 \pm 0.12$ & $450-590 \pm 50$ \\
6 October 1998 & $17: 00-21: 00$ & 5 & 5 & $3.8-7.0$ & $0.41-0.55$ & 0.9947 & $0.56-0.87 \pm 0.44$ & $450-620 \pm 170$ \\
10 October 1998 & $18: 00-21: 00$ & 3 & 7 & $3.3-5.4$ & $0.51-0.71$ & 0.9996 & $0.60-0.67 \pm 0.5$ & $460-530 \pm 280$ \\
14 October 1998 & $16: 00-24: 00$ & 6 & 0.7 & $3.5-6.8$ & $0.46-0.61$ & 0.9997 & $0.64-0.78 \pm 0.07$ & $400-570 \pm 30$ \\
15 October 1998 & $18: 00-24: 00$ & 3 & 3.5 & $3.1-5.4$ & $0.37-0.76$ & 0.9985 & $0.59-0.69^{ \pm} 0.25$ & $620-760 \pm 190$ \\
26 October 1998 & $16: 00-22: 00$ & 4 & 4 & $3.0-5.9$ & $0.57-0.87$ & 0.9997 & $0.62-0.67 \pm 0.15$ & $360-500 \pm 50$ \\
30 October 1998 & $15: 00-22: 00$ & 5 & 1 & $3.0-7.0$ & $0.41-1.11$ & 0.9992 & $0.46-1.02 \pm 0.14$ & $330-440 \pm 40$ \\
\hline
\end{tabular}

The stages of analysis for each time interval are illustrated in Fig. 9, for 4 October 1998. Panel a) shows the cursor clicked determinations of the harmonic frequencies with time for each polarization. The $\mathrm{H}, \mathrm{D}, \mathrm{RH}$ and $\mathrm{LH}$ polarization components are plotted in red, yellow, green and blue respectively. Panel b) shows the diurnal variation in the $f_{0} F 2$ critical frequency from the ionosonde at Sodankylä in red diamonds. Where data is missing or uncertain (most commonly due to absorption in a lower ionospheric layer), the data points are linearly interpolated. For data gaps lasting longer than $5 \mathrm{~h}$, linear interpolation is meaningless and the critical frequency is plotted as 0 . The error associated with the critical frequency is $5 \%$ where the uncertainty is quantified on the ionosonde data tables, and assumed to be about $10 \%$ for linearly interpolated points. The terminator crossings at ground level and at $100 \mathrm{~km}$ altitude above Sodankylä are shown as black and purple dashed lines respectively. The least squares regression lines of the cursor points for each harmonic using all the polarizations are plotted as solid black lines, and their uncertainty (given by the standard deviation of the points) are shown in panel c) as dot-dash lines. On some days, the magnetic field strength of the resonance features vary from one polarization to another, thus the least squares regression lines (see panel c)) through the cursor points (in panels a)) are calculated from less than 4 polarizations. This increases the standard deviation of the points about the least squares regression lines, making the experimental error larger in determining the positions of the resonance features. Panel d) is a plot of how the harmonic spacing, $\Delta f$, varies with time, calculated from the linear functions that describe the harmonic frequency variation with time plotted in panel c).
Scatter plots of the harmonic frequencies $(f)$ versus harmonic number $(N)$ were plotted for each hour when the resonance features were identified. An example of this is given in panel e) of Fig. 9, with error bars that represent the uncertainty in frequency shown in panel c). Least squares regression lines through these points are plotted in different colours for each hour going from purple-yellow with increasing time. For all of the dates analyzed, all of the gradients of the lines increase with time except those during the postmidnight time interval, which decrease. The same is true for the intercepts, although the flatness of the resonance features observed on 6 October 1998 made any variation in intercept very small. The product moment correlation coefficients $(r)$ for $f$ versus $N$ at any instant in time vary, but they are generally very high (the mean values of $r$ during each time interval analyzed are shown in column 7 of Table 1). For example on 14 October 1998 the average value of $r$ is 0.9997, indicating that there is a near perfect linear relationship between harmonic frequency and harmonic number throughout the resonance feature time intervals analyzed.

The strong linear relationships between harmonic frequency and harmonic number for all of the time intervals that were studied are consistent with a homogeneous cavity model used by Trakhtengerts et al. (2000) for the IAR, which was consequently adopted for interpretation purposes. In this simple model, reflection of Alfvén waves occurs within the top and bottom layers, and standing waves are set up in the middle layer. For resonance of waves with wave speed $v_{A}$ in a uniform cavity of length $L$, the frequency $f$ of harmonic 


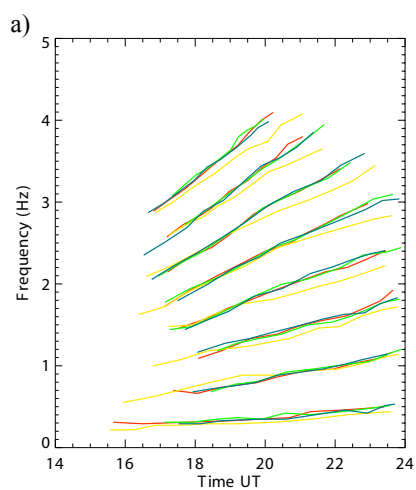

e)

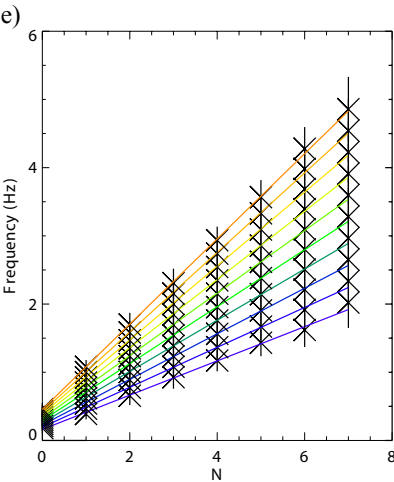

b)

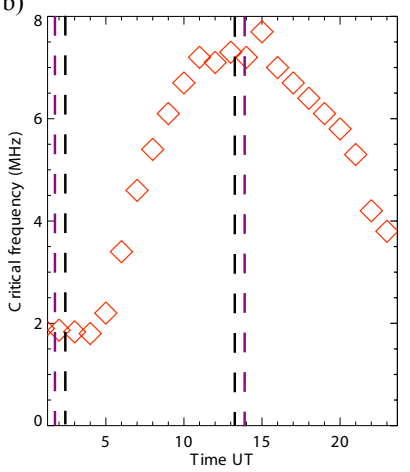

f)

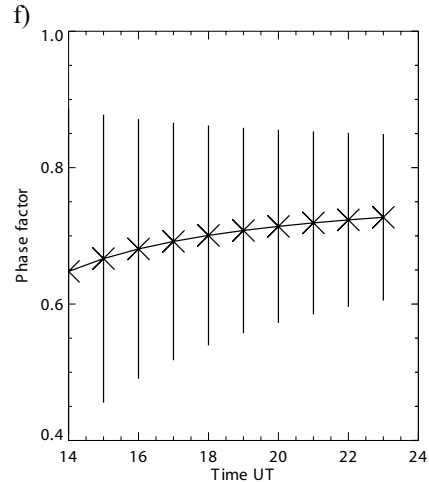

c)

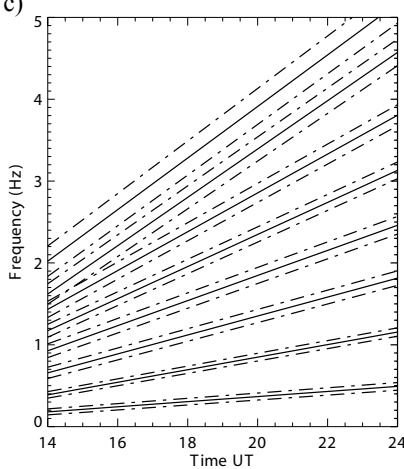

g)

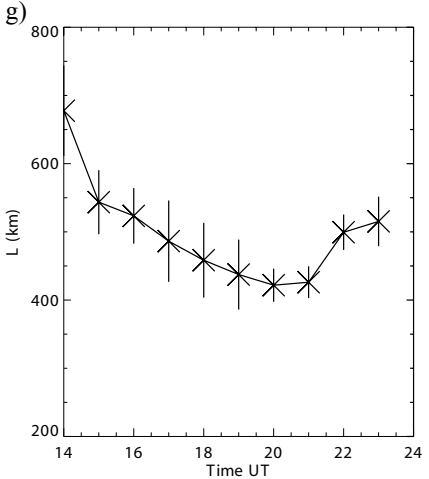

Fig. 9. Analysis of the magnetic spectral resonance features seen in pulsation magnetometer data at Sodankylä, Finland on 4 October 1998 . Panel (a) shows the resonance feature frequency evolution over time derived for each polarisation component: north south component (red), east west (yellow), right-handed circular (green) and left-handed circular polarisation (blue). Panel (b) shows the diurnal $f_{0} F 2$ critical frequency variation from a co-located ionosonde in red diamonds. The terminator crossings at ground level and at 100 km altitude above Sodankylä are shown as black and purple dashed lines respectively. Panel (c) shows least squares regression lines through the harmonic points plotted in panel a), with error margins shown as dot-dashed lines given by the standard deviation of these points. Panel (d) plots the function of harmonic spacing with time, as calculated from the harmonics plotted in panel c). Panel (e) is a scatter plot of harmonic frequency plotted against harmonic number for each hour from 14:00-23:00 UT, with least squares regression lines through these points going purple-yellow with increasing time. Panels (f) and (g) show the evolution with time of wave phase factor $\Phi$ and effective IAR cavity size, $L$, respectively.

number $N$ is given by (Polyakov and Rapoport, 1981):

$f=\frac{(N+\Phi) v_{A}}{2 L}$

where $\Phi$ is the wave "phase factor". This was originally introduced as a constant value of $1 / 4$. (These authors also used $(l+h)$ instead of $L$ in Eq. (1), where $\mathrm{H}$ is the thickness of the bottom side of the ionosphere and $l$ is the scale of the electron density exponential decay in the upper ionosphere.) The value of $\Phi$ may be controlled by the reflection coefficient at the lower IAR boundary, $R_{\text {lower }}$, which can be written in terms of $r=\Sigma_{\omega} / \Sigma_{p}$ and is given by (Lysak, 1991):

$R_{\text {lower }}=\frac{r-1}{r+1}$

where $\Sigma_{\omega}$ the wave conductance $\Sigma_{\omega}=1 / \mu_{0} v_{I A R}$ and $\Sigma_{p}$ is the integrated Pedersen conductivity. If there is a wave node (for high ionospheric conductivity) at the lower boundary of the IAR with the reflection coefficient $R_{\text {lower }} \sim-1$, we expect the value of $\Phi$ to be half integer. For a wave antinode (low ionospheric conductivity) with $R_{\text {lower }} \sim 1, \Phi$ will be integer (Lysak, 1991). There is always an antinode at the upper IAR boundary. The high and low conductivity cases are illustrated in panels a) and b) respectively of Fig. 10. This diagram shows the first 3 IAR eigenmodes in each case, and how many fractions of a wavelength are set up in the IAR cavity of length $L$.

The gradient of a least squares regression line for $f$ against $N$ at an instant in time gives, according to Eq. (1), $K=v_{A} / 2 L$, and the intercept on the $f$-axis at an instant in time gives $K \phi$. Therefore, the intercept divided by the gradient of the least squares regression line at any instant in time gives $\Phi$. The variation of $\hat{O}$ with time on 4 October 1998 is plotted in panel f) of Fig. 11, with error bars given by the combined uncertainty in the intercepts and slopes of the $f$ against $N$ plot at each point in time (i.e. $(\Delta$ $\left.\Phi / \Phi)^{2}=(\Delta K \Phi / K \Phi)^{2}+(\Delta K / K)^{2}\right)$. Column 8 of Table 1 shows the range of values taken by $\Phi$ for each time interval studied and the mean error in these values. The values of $\Phi$ obtained in this study ranged between $1 / 2$ and 1 which was expected from theory, if $\Phi$ represents the fraction of a 
quarter wavelength that forms a standing wave in addition to the integer number of half wavelengths necessary for cavity resonance. Thus $\Phi$ acted as a consistency check on the model.

The average value of $\Phi$ from all of the time intervals analyzed is 0.61 , which is close to the value expected for high conductivity at the lower boundary. The highest value for $\Phi$ of $1.02 \pm 0.26$ occurred on 30 October 1998 which dropped more dramatically than for any other time interval, ending in the lowest value for $\Phi: 0.46 \pm 0.10$. During this interval the $f_{0} F 2$ frequency underwent the largest change, dropping from $7 \mathrm{MHz}$ to $3 \mathrm{MHz}$, and the $f_{O} E$ dropped more than most intervals, from $3.5 \mathrm{MHz}$ to $1.75 \mathrm{MHz}$. This means that the plasma density in the $\mathrm{E}$ and $\mathrm{F}$ regions dropped, reducing the Pedersen conductivity. However, from the analysis, $\Phi$ went from integer (expected for low conductivity) to half integer (high conductivity), counter to what is expected from theory. Preliminary comparisons of the lower boundary reflection coefficient (calculated using electron density and temperature data from the EISCAT incoherent scatter radar) on 2 days in October 1998 did not show a consistent picture either. Thus the value of $\Phi$ obtained with this simple modelling interpretation of IAR resonance features cannot be used to infer the $\mathrm{E}$ region conductivity.

The variation of $L$ with time can also be calculated if the variation of $v_{A}$ with time is known. This is provided by hourly estimates of the $f_{0} F 2$ critical frequency from the ionosonde at Sodankylä, which measures the peak plasma density in the F2-layer, and so determines how the density in the IAR varies in time, and hence how the Alfvén speed $v_{A}$ in the IAR varies with time. Since $K$ is a function of $v_{A}$ and $L$, the variation of $L$ with time is given by $v_{A} / 2 K . v_{A}$ was calculated from the $f_{0} F 2$ critical frequency (in $\mathrm{MHz}$ ) since ionosondes measure the electron number density $(\mathrm{Ne})$ of the F2 layer directly $\left(N e=1.24 \times 10^{10} \times f_{0} F 2^{2}\right)$ :

$v_{A}=\frac{B}{f_{O} F 2 \sqrt{1.24 \times 10^{10} \mu_{0} m_{i}}}$

where the average mass loading of the field line in the IAR, $m_{i}$ is assumed to be 24.7 a.m.u. (calculated using the IRI model, taking and average over the height range of 100$300 \mathrm{~km}$ altitude with appropriate ionospheric conditions input) and $B$ is the average geomagnetic field strength at these altitudes. This is expected to yield underestimated values of $L$, because the effective IAR density that affects the trapped Alfvén waves is smaller than the density of the F2 peak. The calculated variations in $L$ (shown in panel $\mathrm{g}$ ) for 4 October 1998) simply indicate the variation in the scale size of the IAR with time. The error bars shown in panel g) are given by the combined uncertainty in the gradients of the $f$ against $N$ plots and the $f_{0} F 2$ frequency at each point in time (i.e. $\left.(\Delta L / L)^{2}=(\Delta K / K)^{2}+\left(\Delta f_{O} F 2 / f_{O} F 2\right)^{2}\right)$.

Column 9 of Table 1 summarises the variation of $L$ for each time interval studied. The average value of $L$ from all of the time intervals is $530 \mathrm{~km}$. The maximum variation of $L$ occurred in the morning time interval of 4 October 1998, with $L$ increasing from $700-1140 \pm 100 \mathrm{~km}$. The minimum
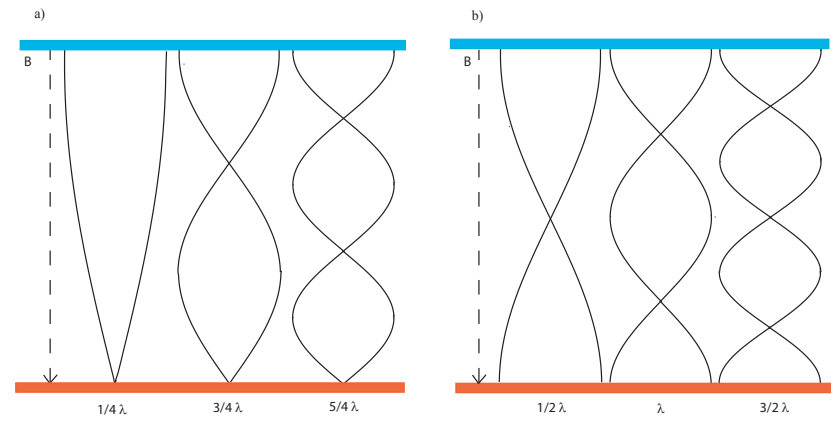

Fig. 10. Diagram showing an Alfvén wave's perpendicular electric field for the first 3 eigenmodes trapped in the IAR for the high conductivity ionosphere (panel (a)) and low conductivity (panel (b)) ionosphere. The lower red rectangles represent the lower IAR boundary and the blue rectangle represents the upper IAR boundary. The background magnetic field is directed downwards for the high latitude northern hemisphere regions. Panel a) for a high conductivity ionosphere shows a node at the lower IAR boundary. Panel b) for a low conductivity ionosphere displays an antinode at the lower IAR boundary.

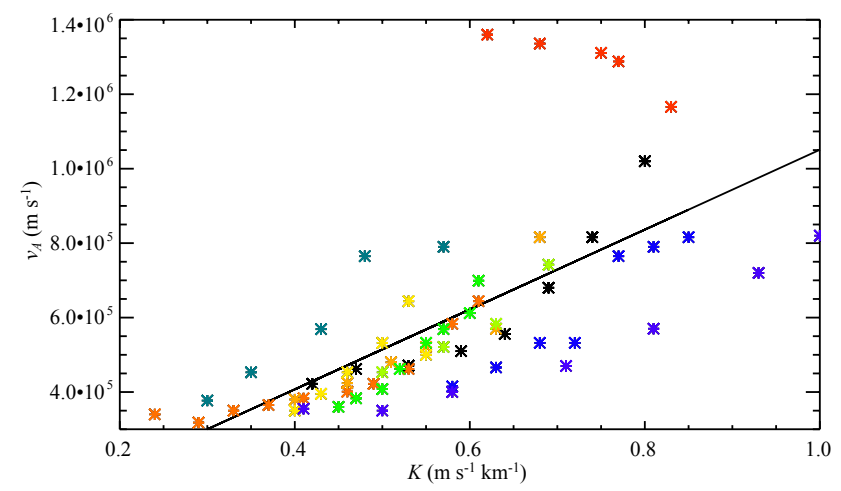

Fig. 11. Scatter plot of the IAR resonance feature analysis from Sodankylä during October 1998, where the colour of the stars vary according to date, going from red to blue with chronological order. On the horizontal axis is plotted gradient, $K$, of the lines of best fit of $f$ against $N$ at each hour for the IAR resonance features. The vertical axis is Alfvén velocity calculated directly from the electron density given by the $f_{O} F 2$ hourly data, assuming that the mean ion mass in the IAR is given by the mean in the F region $\sim 24.7$ a.m.u. The gradient of the least squares regression line through these points gives $2 L$, where $L=530 \mathrm{~km}$, the mean IAR scale height for all of the 10 intervals of IAR resonances that were analysed in detail.

variation occurred on 10 October 1998, although the error margin is large for this day; $L$ varies from $460-530 \pm 280 \mathrm{~km}$. In summary, a typical scale size for the IAR is $\sim 500 \mathrm{~km}$, which is $2-3$ times the typical plasma scale height in the ionosphere.

These results for $L$ can also be explained with the aid of the scatter diagram shown in Fig. 11. The gradients, $K$, of all of the hourly lines of best fit of $f$ against $N$ are plotted against the hourly values for $v_{A}$ calculated directly from the $f_{0} F 2$ critical frequencies and assuming that 
$m_{i}=24.7$ a.m.u. The different coloured stars symbolize data for different days. The gradient of the least squares regression line through all of the points gives $2 L$, where $L=530 \mathrm{~km} \pm 150 \mathrm{~km}$, the average scale size and standard deviation of the IAR respectively for all 10 time intervals of IAR resonance features studied. The red stars represent data for the sole post-midnight interval (just after dawn) on 4 October 1998 when the $f_{0} F 2$ frequency rapidly increased, hence making the IAR size increase rapidly (as described above).

This study of IAR resonance features has shown that they are commonly observed in the quiet-time background noise from the Sodankylä pulsation magnetometer; in October 1998 multiple harmonics were observable for about $66 \mathrm{~h}$, most commonly after 16:00 UT which is $1-2 \mathrm{~h}$ after sunset, and sometimes into the early hours of the morning, $1-2 \mathrm{~h}$ after sunrise. The longest interval of resonance features lasted for $13 \mathrm{~h}$ from 16:00 UT on 3 October 1998 until 05:00 UT on 4 October 1998. The harmonics are quasi-evenly spaced at all times, as shown from the strong linear relationships between harmonic frequency and harmonic number.

The frequency of each harmonic varies with time of day very slowly and systematically, on average the harmonic spacing $c$ increased by $\sim 0.06 \mathrm{~Hz}$ per hour towards midnight and decreased by $0.02 \mathrm{~Hz}$ per hour post midnight. The relative changes in the fundamental frequency over the time intervals were calculated, with an average change over all the time intervals of $52 \%$. The relative change in the effective cavity size, $L$ was calculated; whose average is $32 \%$ over a time interval of a few hours. This is low compared to the relative change in $v_{A}$ of $61 \%$ due to the diurnal variation of the plasma density in the F2 layer. This indicates that the diurnal evolution of the IAR harmonic frequencies is chiefly caused by the changing Alfvén velocity in the F2 region of the ionosphere. The Alfvén velocity is proportional to $f_{O} F 2^{-1}$, so as the $f_{O} F 2$ frequency drops rapidly towards midnight and rises more slowly in the morning (see Fig. 9 panel b)) the IAR spectral resonance features do the converse. These results support the results published by Belyaev et al. (1990) who used data from a mid-latitude magnetometer station and an ionosonde. It was also demonstrated qualitatively by Belyaev et al. (1999) using 2 days of data from a high-latitude magnetometer and electron densities variations derived from EISCAT incoherent scatter radar data. However it is not only the Alfvén velocity that determines the evolution of the IAR eigenfrequencies but also the size of the IAR cavity, $L$, as discussed by Odzimek, (2004). Yahnin et al. (2003) also calculated the long-term variations of a parameter they termed $L$ characterizing the scale of electron density decrease above the F-layer maximum at Sodankylä similar to $L$ in this paper but without estimating the average ion mass $m_{i}$ in the IAR. They used ionosonde $f_{0} F 2$ measurements and SRS frequency interval $\Delta f$ from magnetometer data averaged over 3-h intervals. The parameter showed seasonal variability, peaking in summer months where data was available for nighttime time intervals. During October 1998 the diurnal variation of $L$ was not more than $30 \%$, which agrees with the results of the present study. Thus, $L$ is a relatively stable parameter; the diurnal evolution of the harmonic frequencies is most strongly dependent on Alfvén velocity, since IAR size is less variable.

The relative change in $\Phi$ calculated for the time intervals of this study was very low, at 20\%. With reference to Eq. (1), the values of $\Phi$ obtained in this study ranged between $1 / 2$ and 1 , thus acted as a consistency check on the model. It is believed that $\Phi$ determines the fraction of a quarter wavelength that forms a standing wave in addition to the integer number of half wavelengths necessary for cavity resonance. The value of $\Phi$ depends on whether the wave reflection at the lower boundary is a node or an antinode, or something in between. A preliminary comparison of the diurnal variation of $\Phi$ and the ionospheric conductivity/lower boundary reflection coefficient was made using $3 \mathrm{~h}$ of EISCAT incoherent scatter radar data available during October 1998 when IAR resonance features were observed. However, these results were not conclusive and further investigation is necessary on other days when IAR resonance features and radar data coincide.

\section{Conclusion}

Spectral IAR resonance features were successfully identified in pulsation magnetometer data from Sodankylä, Finland on 13 out of 30 days in October 1998. The variations in the resonance frequencies over time were quantified and analysed in detail for 10 days, using dynamic colour spectra of the data and an interactive cursor clicking technique. Strong linear relationships between harmonic frequency and harmonic number for all of the time intervals studied enabled a homogeneous cavity model for the IAR to be adopted to analyze the data further. When the variation of the $f_{0} F 2$ region at the same location was taken into account, the detailed analysis of magnetic resonance features yielded a parameter that describes the effective size of the IAR, $L$. During the time intervals studied, the average variation of $L$ over the time intervals studied was $32 \%$, whereas the Alfvén velocity calculated electron density in the $\mathrm{F} 2$ region of the ionosphere varied by $61 \%$. Thus the IAR cavity is fairly stable over the $3-10 \mathrm{~h}$ time intervals studied and over the 30 day interval over which IAR resonance features were analyzed during October 1998. Therefore the diurnal evolution of the IAR eigenfrequencies is caused, in the main, by the changing density of the IAR throughout the day, confirming previous studies of IAR resonance features and local IAR parameters (Demekhov, 2000, Odzimek, 2004, Yahnin et al, 2003). Another IAR parameter was derived from the analysis of the IAR resonance features associated with the phase matching structure of the standing waves in the IAR, termed the phase factor, $\Phi . \Phi$ varied over the time intervals studied by $20 \%$ on average, possibly due to changing ionospheric conductivity, although the results were inconclusive due to a lack of incoherent radar data when IAR resonance features are observed. The value of $\Phi$ is thought to depend on whether the wave reflection at the 
lower boundary is a node or an antinode, or something in between. Further investigation is necessary on other days when IAR resonance features and radar data coincide to determine the relationship between $\Phi$ and the lower IAR boundary reflection coefficient.

Acknowledgements. The Editor in chief thanks A. Yahnin and another referee for their help in evaluating this paper.

\section{References}

Belyaev, P. P., Bosinger, T., Isaev, S. V., and Kangas, J.: First evidence at high latitudes for the Ionospheric Alfvén resonator, J. Geophys. Res., 104(A3), 4305-4317, 1999.

Belyaev, P. P., Polyakov, S. V., Rapoport, V. O., and Trakhtengerts, V. Yu.: Discovery of resonance structure in the spectrum of atmospheric electromagnetic background noise in the range of shortperiod geomagnetic pulsations, Doklady Akademii Nauk SSSR, 297, 840-846, 1987.

Belyaev, P. P., Polyakov, S. V., Rapoport, V. O., and Trakhtengerts, V. Yu.: Izu. VUZov, Radiofizika, XXXII(N6), 1989.

Belyaev, P. P., Polyakov, S. V., Rapoport, V. O., and Trakhtengerts, V. Yu.: The Ionospheric Alfvén resonator, J. Atmos. Terr. Phys., 52, 781-788, 1990.

Belyaev, P. P., Polyakov, S. V., Ermakova, E. N., and Isaev, S. V.: Solar cycle variations in the ionospheric Alfvén resonator 19851995, J. Atmos. Terr. Phys., 62, 239-248, 2000.

Carlson, C. W., Pfaff, R. F., and Watzin, J. G.: The Fast Auroral SnapshoT mission, Geophys., Res., Lett., 25(12), 2013-2016, 1998.

Cash, S. R., Davies, J. A., Kolesnikova, E., Robinson, T. R., Wright, D. M., Yeoman, T. K., and Strangeway, R. J.: Electron acceleration observed by the FAST satellite within the IAR during a $3 \mathrm{~Hz}$ modulated EISCAT heater experiment, Ann. Geophys., 20, 1499-1507, 2002,

\section{SRef-ID: 1432-0576/ag/2002-20-1499.}

Demekhov, A. G., Belyaev, P. P., Isaev, S. V., Manninen, J., Turunen, T., and Kangas, J.: Modelling the diurnal evolution of the resonance spectral structure of the atmospheric noise background in the Pc1 frequency range, J. Atmos. Terr. Phys., 62, 257-265, 2000.

Hickey, K., Sentman, D. D., and Heavner, M. J.: Ground-based observations of ionospheric Alfvén resonator bands, Fall meeting AGU, A22C-08, 1996.
Kolesnikova, E., Robinson, T. R., Davies, J. A., Wright, D. M., and Lester, M.: Excitation of Alfvén waves by modulated HF heating of the ionosphere, with application to FAST observations, Ann. Geophys., 20, 57-67, 2002,

SRef-ID: 1432-0576/ag/2002-20-57.

Lynn, P. A.: An Introduction to the Analysis and Processing of Signals, Macmillan, 1984.

Lysak, R. L.: Feedback instability of the Ionospheric Resonant cavity, J. Geophys. Res., 96(A2), 1553-1568, 1991

Odzimek, A.: Numerical estimate of the spectral resonance structure frequency scale of natural ULF magnetic field, Stud. Geophys. Geod., 48, 647-660, 2004.

Polyakov, S. V.: On properties of an ionospheric Alfvén resonator, in Symposium KAPG on Solar-Terrestrial Physics, vol. III, 7273, Nauka, Moscow, 1976.

Polyakov, S. V. and Rapoport, V. O.: Ionospheric Alfvén resonator, Geomagnetism and Aeronomy, 21 (5), 816-822, 1981.

Rishbeth, H., and Williams, P. J. S.: The EISCAT Ionospheric Radar: the System and its Early Results, Q. Jl. R. astr. Soc., 26, 478-512, 1985.

Robinson, T. R., Strangeway, R. J., Wright, D. M., Davies, J. A., Horne, R. B., Yeoman, T. K., Stocker, A. J., Lester, M., Rietveld, M. T., Mann, I. R., Carlson, C. W., and McFadden, J. P.: FAST observations of ULF waves injected into the magnetosphere by means of modulated RF heating of the auroral electrojet, Geophys. Res. Lett., 27, 3165-3168, 2000.

Stubbe, P., Kopka, H., Lauche, H., Reitveld, M. T., Brekke, A., Holt, O., Jones, T. B., Robinson, T. R., Hedberg, A., Thide, B., Crochet, M., and Lotz, H. J.: Ionospheric modification experiments in northern Scandinavia, J. Atmos. Terr. Phys., 44, 10251041, 1982.

Trakhtengertz, V. Y., Belyaev P. P., Polyakov, S. V., Demekhov, A. G., and Bosinger, T.: Excitation of Alfvén waves and vortices in the ionospheric Alfvén resonator by modulated powerful radio waves, J. Atmos. Terr. Phys., 62, 267-276, 2000.

Wright, D. M., Davies, J. A., Yeoman, T. K., Robinson, T. R, Cash, S. R., Kolesnikova, E., Lester, M., Chapman, P. J., Strangeway, R. J., Horne, R. B., Rietveld, M. T., and Carlson, C. W.: Detection of artificially generated ULF waves by the FAST spacecraft and its application to the "tagging" of narrow fluw tubes, J. Geophys. Res., 108(A2), 1090, 2003.

Yahnin, A. G., Semenova, N. V., Ostapenko, A. A., Kangas, J., Manninen, J., and Turunen, T.: Morphology of the spectral resonance structure of the electromagnetic background noise in the range of $0.1-4 \mathrm{~Hz}$ at $\mathrm{L}=5.2$, Ann. Geophys., 21, 779-786, 2003, SRef-ID: 1432-0576/ag/2003-21-779. 